\title{
PHYSICS OF DENSE MATTER
}


This page is intentionally left blank 


\title{
PHYSICS OF DENSE MATTER
}

\author{
Y. C. Leung \\ Professor of Physics \\ Southeastern Massachusetts University
}

\section{Science Press}

Beijing, China

\section{World Scientific}

Singapore 


\title{
Published by
}

Science Press

Beijing China
World Scientific Publishing Co. Pte. Ltd.

P. O. Box 128, Farrer Road, Singapore 9128

Responsible Editor: Zhang Banggu

\begin{abstract}
Copyright $\odot 1984$ by Science Press and World Scientific Publishing Co. Pte. Ltd. All rights reserved. This book or parts thereof, may not be reproduced in any form or by any means, electronic or mechanical, including photocopying, recording or any information storage and retrieval system now known or to be invented, without written permission from the Publisher.
\end{abstract}

ISBN $9971-978-10-5$

Science Press Book No. 3445-42

Printed by C \& C Joint Printing Co., (H.K.) Ltd. 


\section{To My Parents}


This page is intentionally left blank 


\section{PREFACE}

This book grew from lecture notes prepared for a ten-week summer short-course on dense matter physics that I gave to a group of students and physicists at the Beijing Normal University (China) in 1979. My aim was to introduce the field of dense matter physics which saw rapid development in the past ten to fifteen years to an audience who was not yet active in the field. I wanted to make the subject matter as simple and yet as complete as I could by concentrating on only a few well-developed topics, and I tried to spell out all details as clearly as possible. My hope was to provide enough background information on these topics that the participants of the short-course might be prepared to comprehend the current research articles. In reality, I was far from reaching my goal, and so in 1981 when I was approached by the Science Press to publish the lecture notes, I decided to rewrite them. The final result comprises the present book, which contains fewer topics but more detailed and complete description of each.

This book is intended to prepare the readers for theoretical studies in dense matter physics. It may also be used pedagogically as an introduction to the manybody theory with emphasis on illustrative examples. Many mathematical operations needed for this field are carried out in details, usually not in the most elegant way but in a practitioner's way, so that readers who are handicapped in background training can still follow. Hopefully this book will enable more newcomers to enter into the study of dense matter.

I wish to thank my colleagues at the Beijing Normal University for comments and helpful suggestions on the contents of this book. Among them I wish to mention the following persons: Liang Shao-rong, Li Zong-wei, Gao Shang-hui, Ge Yun-zao and Shi Tian-yi, whom I had the pleasure to work with during the preparation of this book. Finally, I wish to thank Chen Chung-kuang of the Graduate School of the Academy of Sciences for arranging with the Science Press for me to publish this book.

Y.C. Leung

N. Dartmouth, Massachusetts

May 4, 1983 
This page is intentionally left blank 


\section{CONTENTS}

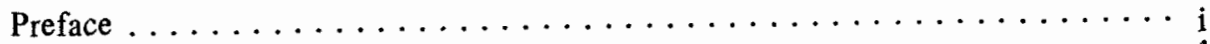

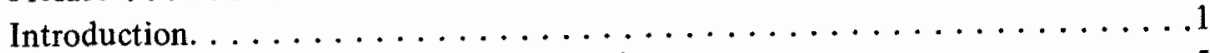

Chapter 1. Regime 1: $500<\rho<10^{8} \mathrm{~g} / \mathrm{cm}^{3}$. Subferrous Matter. ........

1. Variational Methods. . . . . . . . . . . . . . . . . .

o Hartree equations o Hartree-Fock equations

2 Ideal Degenerate Electron Gas . . . . . . . . . . . . . 14

o Quatnum states o pressure o equation of state

3. Electrostatic Interaction . . . . . . . . . . . . . . . 26

o Thomas-Fermi method o Thomas-Fermi-Dirac method

4. Thermal Effects . ........................40

o Partition function o entropy ofree energies

o thermal Thomas-Fermi method o thermal Hartree-Fock method

Chapter 2. Regime 2: $10^{8}<p<10^{12} \mathrm{~g} / \mathrm{cm}^{3}$. Subnuclear Matter. ........52

5. Neutronization Process. . . . . . . . . . . . . . . . . 52

o chemical equilibrium

6. Nuclear Semi-empirical Mass Formula and Nuclear Mass Tables . . . . . . 57

o nuclear saturation o nuclear binding energy

o lattice energy

7. Nuclear Two-Body Interactions $\ldots \ldots \ldots \ldots \ldots \ldots \ldots \ldots \ldots . \ldots 70$

o nuclear spin o charge independence o static potentials

8. Nuclear Effective Interactions .....................83

o Skyrme potentials o direct and exchange interactions

o nuclear compressibility

9. Finite Nuclei ...............

o orbital wave functions o Coulomb interaction

o Hartree-Fock solutions

10. Subnuclear Matter at Finite Temperatures ...............114

o thermal Hartree-Fock solutions o two-phase bulk equilibrium

o surface energy

Chapter 3. Regime 3:10 $12<\rho<10^{15} \mathrm{~g} / \mathrm{cm}^{3}$. Nuclear and Transnuclear

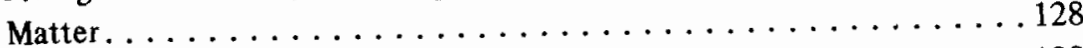

11. Nucleon-Nucleon Scattering and Realistic Nuclear Potentials . . . . . . 128 
o Lippmann-Schwinger equation o partial wave decompositions

o vector spherical harmonics o Reid potentials

12. Independent-Pair Model of Nuclear Structure .

o two-nucleon correlations o Brueckner-Bethe-Goldstone equation

o reaction matrix o defect wave function o neutron matter

13. The Constrained Variational Method. . . . . . . . . . . . . . 163

o correlation factors o projection operators for coupled states

o Lowest Order Constrained Variational equations

14. Relativistic Models.

o Dirac equation o relativistic Hartree-Fock equation

Chapter 4. Regime 4: $\rho>10^{16} \mathrm{~g} / \mathrm{cm}^{3}$. Ultradense Matter. . . . . . . . . 195

15. Meson Theories . . . . . . . . . . . . . . . . . . . . . . . . . . . 195

o field operators o Lagrangian o retarded Green's function

o mean field model

16. Baryonic Matter . . . . . . . . . . . . . . . . . . . . . . . . 213

o hyperon production thresholds o hyperon interactions

17. Pion Condensation. . . . . . . . . . . . . . . . . 221

o pion-nucleon interaction o condensation threshold

18. Quark Matter . . . . . . . . . . . . . . . . . . . 234

- QCD o bag model o equation of state

Appendix A. Physical Constants and Astronomical Parameters . . . . . . . 245

Appendix B. Coordinates and Special Functions. . . . . . . . . . . . . . . 246

B.1 Spherical Polar Coordinates . . . . . . . . . . . . . . 246

B.2 Spherical Harmonics . . . . . . . . . . . . . . . . . . 248

B.3 Bessel Functions and Spherical Bessel Functions. . . . . . . . . 250

Appendix C. Equation of State of Dense Matter . . . . . . . . . . . . . 252

Exercises . . . . . . . . . . . . . . . . . . . . . . . . . 258

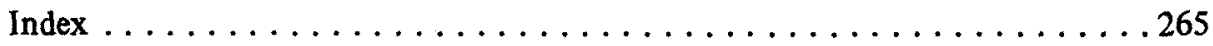

